\title{
Avaliação de um sistema automatizado na identificação de espécies de Enterococcus
}

\section{Evaluation of an automated system for the identification of Enterococci}

Pedro Alves d'Azevedo'; Vladimir Cantarelli²; Everton Inamine²; Silvana Superti³; Cícero Armídio Gomes Dias ${ }^{1,3}$

Unitermos
Identificação
automatizada
Enterococos
E. faecalis

\section{resumo}

O uso de métodos automatizados tem freqüentemente levado a falhas na identificação do gênero Enterococcus em laboratórios de microbiologia clínica. Neste estudo foi avaliada a utilização de um sistema automatizado Vitek (bioMérieux) em dois laboratórios de microbiologia clínica para identificação de diferentes espécies de enterococos. Os resultados foram comparados com os testes fisiológicos convencionais. As amostras (80) foram inoculadas em testes bioquímicos convencionais e no cartão Vitek GPI. No geral, a concordância entre os dois métodos foi de 83,7\% (67/80). Entre as amostras de E. faecalis, o sistema Vitek identificou corretamente $35 / 40(87,5 \%)$ e entre os E. faecium, a concordância foi $12 / 14$ (85,7\%). Em 20/26 amostras (76,9\%) pertencentes a espécies não-E. faecalis e não-E. faecium, o sistema chegou à identificação correta. Os resultados do presente estudo mostram que o sistema Vitek necessita de melhorias para a identificação de enterococos, especialmente diante de espécies menos freqüentes.

abstract

Automated systems may present problems in the identification of members of the genus Enterococcus in clinical laboratories. Having conventional physiological tests as the reference method, we evaluated the use of an automated system (VITEK - bioMérieux) in the identification of 80 isolates belonging to different species of Enterococcus. The general agreement between results obtained by the conventional method and by the Vitek GPI card was $83.7 \%$ (67/80). Among isolates of E. faecalis and E faecium we observed that the automated system correctly identified $35 / 40$ (87.5\%) and 12/14 (85.7\%) of the strains, respectively. Among isolates belonging to species which are neither E. faecalis, nor E. faecium, it was observed an agreement of 20/26 (76.9\%). Results point to the need of improvement in the automated systems to identify enterococci. Special consideration must be taken regarding less frequently isolated species. key words

Automated identification

Enterococci

E. faecalis 


\section{Introdução}

A importância dos enterococos como patógeno nosocomial tem sido bem documentada nos últimos anos ${ }^{(4,7}$, 12). Enterococcus faecalis é a espécie mais freqüentemente associada com infecções em humanos, seguida pelo Enterococcus faecium ${ }^{(2)}$.

As espécies de enterococos podem ser identificadas rotineiramente através de esquemas, empregando testes convencionais baseados em características fenotípicas que permitem separar estes microrganismos em cinco grupos fisiologicamente diferentes ${ }^{(3)}$.

Cada vez mais, laboratórios clínicos têm utilizado sistemas automatizados para identificar microrganismos de uma forma geral. Para a identificação de enterococos, os mais utilizados são o Vitek, o MicroScan e o Pasco(5,6,11). Entretanto, em várias situações, seu uso leva à obtenção de identificações errôneas que necessitam ser esclarecidas através do uso de métodos convencionais e da análise dos perfis de proteínas totais, a qual demonstrou ser um método reprodutível e válido para diferenciar as espécies de enterococos ${ }^{(3,9)}$.

O objetivo deste estudo foi o de comparar os resultados obtidos do sistema automatizado Vitek cartão GPI (bioMérieux Vitek Inc., Hazelwood, Mich.) com os resultados de técnicas convencionais para identificação de diferentes espécies de enterococos.

\section{Material e método}

\section{Amostras bacterianas}

Um total de 80 amostras bacterianas foi incluído neste estudo. As amostras foram obtidas da nossa coleção, localizada no Laboratório de Cocos Gram positivos da Fundação Faculdade Federal de Ciências Médicas de Porto Alegre, e estavam mantidas sob a forma de suspensões em solução contendo leite desnatado Molico (Nestlé, Araçatuba-SP, Brasil) a $10 \%(\mathrm{p} / \mathrm{v})$ e glicerol a $10 \%(\mathrm{v} / \mathrm{v})$, mantidas congeladas a $20^{\circ} \mathrm{C}$ negativos. As espécies de enterococos, identificadas de acordo com os resultados do esquema de identificação convencional foram: Enterococcus faecalis ( $n=40)$, Enterococcus faecium $(n=14)$, Enterococcus raffinosus ( $n=2)$, Enterococcus avium $(n=5)$, Enterococcus gallinarum ( $n=9)$, Enterococcus durans $(n=3)$, Enterococcus casseliflavus $(n=2)$, Enterococcus hirae $(n=4)$ e Lactococcus garvieae $(n=1)$. Neste grupo foram inseridas as seguintes amostras-padrão: E. faecalis SS-1273, E. faecium-1274, E. gallinarum SS-1228, E. casseliflavus SS-1229, E. avium SS-559, E. durans SS-661 e E. hirae SS-1227.

\section{Identificação das amostras bacterianas por métodos de referência e método automatizado}

A pureza de cada amostra foi confirmada após cultivo em Brain Heart Infunsion (BHI) ágar, (DIFCO Laboratories, Detroit-MI, EUA) acrescido de $5 \%(\mathrm{v} / \mathrm{v})$ de sangue desfibrinado de carneiro e incubado por $24 \mathrm{~h}$ a $35^{\circ} \mathrm{C}$. Após o período de incubação, as amostras foram submetidas aos testes bioquímicos convencionais que incluíam detecção da formação de ácidos a partir de diferentes carboidratos (manitol, sorbose, arabinose, sorbitol, rafinose e sacarose) e hidrólise (ou desaminação) da arginina; tolerância ao telurito; motilidade; produção de pigmento; e utilização do piruvato, bem como o uso de testes complementares ${ }^{(1,}$ 2, 3). Algumas amostras também foram identificadas pela análise do seu perfil de proteínas totais, seguindo as recomendações de Merquior et al para confirmar os resultados discordantes entre as duas metodologias empregadas, já que o perfil de proteínas totais é espécie-específico no gênero Enterococcus ${ }^{(2)}$. O sistema automatizado Vitek (bioMérieux) foi avaliado através da inoculação das amostras, e utilizou o cartão Vitek GPI (bioMérieux), sendo seguidas as instruções do fabricante quanto a preparo de inóculo, incubação, leitura e interpretação.

\section{Resultados e discussão}

Os resultados obtidos no sistema automatizado Vitek foram comparados com os testes fisiológicos convencionais (Tabela). No geral, a concordância nas identificações entre os dois sistemas foi de $83,7 \%(67 / 80)$.

Entre as amostras de Enterococcus faecalis, o sistema Vitek identificou corretamente $35 / 40(87,5 \%)$, sendo as demais amostras identificadas como Enterococcus avium (duas amostras), Enterococcus faecium (uma amostra) e

Comparação entre a identificação de Enterococcus através do cartão GPI

Tabela Vitek e do método de referência*

Espécie Número de amostras em concordância Método de referência Sistema Vitek

\begin{tabular}{llll} 
& & $(\mathrm{N})$ & $(\%)$ \\
\hline E. faecalis & 40 & 35 & 87,5 \\
E. faecium & 14 & 12 & 85,7 \\
Não-E. faecium & 26 & 20 & 76,9 \\
$\quad$ e não-E. faecalis & & & \\
\hline
\end{tabular}

*Método de referência: testes convencionais segundo Facklam, Sahm \& Teixeira (1999). 
Enterococcus gallinarum (uma amostra), e outra amostra não foi identificada.

Nas amostras de Enterococcus faecium, a concordância foi verificada em 12/14 (85,7\%), havendo duas identificações incorretas pelo sistema Vitek, ambas identificadas como Enterococcus gallinarum.

Em 20/26 (76,9\%) amostras pertencentes às espécies não-Enterococcus faecalis e não-Enterococcus faecium, o sistema chegou à identificação correta.

Todas as amostras discordantes entre o método convencional e o sistema automatizado foram confirmadas através da análise do perfil de proteínas totais, que é espécieespecífico no gênero Enterococcus ${ }^{(9)}$, demonstrando assim que as amostras incluídas neste estudo não apresentavam nenhuma atipia fenotípica.

O sistema Vitek apresentou boa performance com relação à identificação de E. faecalis e E. faecium. As demais espécies apresentaram problemas de identificação em cerca de $23 \%$ das cepas testadas. As principais falhas obtidas no cartão Vitek GPI foram na leitura dos carboidratos arabinose e sorbitol e na descarboxilação da arginina em todas as espécies. Os resultados discordantes do cartão GPI obtidos em relação às espécies testadas variaram de 55\% a $99 \%$ em termos de probabilidade. Chama a atenção em um dos laudos uma identificação incorreta pelo sistema, um Enterococcus gallinarum como Enterococcus avium, com 99\% de probabilidade. Outros exemplos de falhas com diferentes porcentuais de probabilidade foram: E. gallinarum identificado como $E$. faecalis, E. faecium identificado como E. gallinarum e E. hirae não-identificado. A amostra de Lactococcus garviae foi incluída neste estudo para verificar o seu comportamento frente ao sistema automatizado, já que as suas características fenotípicas são muito semelhantes a algumas espécies do grupo II do gênero Enterococcus, o que dificulta a sua correta identificação no laboratório. Neste trabalho, a amostra foi identificada erroneamente como sendo E. gallinarum.

Os resultados do presente estudo mostram que o sistema Vitek, na versão utilizada, é um sistema que necessita de melhorias para a identificação de enterococos, especialmente diante de espécies menos freqüentes, concordando com outros estudos já realizados anteriormente com amostras de enterococos americanas e européias ${ }^{(8,10)}$. Chamamos a atenção também que a dificuldade no tratamento de infecções, principalmente com relação aos enterococos resistentes à vancomicina, enfatiza a necessidade de uma segura e rápida identificação para orientação terapêutica e conduta de controle de infecção hospitalar.

\section{Referências}

I. FACKLAM, R. R.; COLLINS, M. D. Identification of Enterococcus species isolated from human infections by a conventional test scheme. J Clin Microbiol, v. 27, p. 73 I-4, 1989.

2. FACKLAM, R. R.; SAHM, D; TEIXEIRA, L. M. Enterococcus. In: MURRAY, P. R.; BARON, E. J.; PFALLER, M. A.;TENOVER, F. C.; YOLKEN, R. H.; WASHINGTON, D. C. (Eds.). Manual of clinical microbiology. 7. ed. ASM Press, 1999. p. 297-305.

3. FACKLAM, R. R.; TEIXEIRA, L. M. Enterococcus. In: COLLIER, L.; BALOWS, A.; SUSSMAN, M. (Eds.). Topley and Wilson's microbiology and microbial infections. 9. ed. Edward Arnold. London: United Kingdom, 1997. p. 669-82.

4. GRAY, J.W.; PEDLER, S. J. Antibiotic-resistant enterococci.] Hosp Infect, v. 21 , p. I- 14, 1992

5. IWEN, P.C. et al. Revised approach for identification and detection of ampicillin and vancomycin resistance in Enterococcus species by MicroScan panels. J Clin Microbiol, v. 34, p. 1779 83, 1996.

6. JETT, B.; FREE, L.; SAHM, D. F. Factors influencing the Vitek grampositive susceptibility system's detection of van B encoded vancomycin resistance among enterococci. J Clin Microbiol, v. 34, p.70I-6, 1996

7. LECLERCQ, R. et al. Plasmid-mediated resistance to vancomycin and teicoplanin in Enterococcus faecium. N Eng J Med, v. 319 p. |57-6|, 1988
8. LIASSINE, N. et al. Characterization of glycopeptide-resistant enterococci from a Swiss hospital. J Clin Microbiol, v. 36, p. $1853-8,1998$.

9. MERQUIOR, V. L. C. et al. Analysis of electrophoretic whole-cell protein profiles as a tool for characterization of Enterococcus species. Curr Microbiol, v. 28, p. 149-53, 1994.

10. SADER, H. S. et al. Evaluation of Vitek and API $20 \mathrm{~S}$ for species, identification of enterococci. Diagn Microbiol Infect Dis, v. 22, p. 315-9, 1995

I I.TSAKRIS, A. et al. Apparent increased prevalence of high-level aminoglycoside-resistant Enterococcus durans resulting from false identification by a semiautomated software system. J Clin Microbiol, v. 36, p. 1419-21, 1998.

12. WILLEY, B. M. et al. Detection of vancomycin resistance in Enterococcus species. J Clin Microbiol, v. 30, p. I621 -4, 1992.

Endereço para correspondência

Pedro A. d'Azevedo

Fundação Faculdade Federal de Ciências Médicas de Porto Alegre

Rua Sarmento Leite 245/211

CEP 90050-170 - Porto Alegre-RS

Tel.: (51) 3224-8822

Fax: (51) 3226-9756

e-mail: pedroaze@fffcmpa.tche.br 